\title{
MINIMAL SHELL OF A TYPICAL CONVEX BODY
}

\author{
ANDREANA ZUCCO \\ (Communicated by William J. Davis)
}

\begin{abstract}
We prove that "most" convex bodies in $E^{d}$ touch the boundary of their minimal shell in precisely $d+2$ points.
\end{abstract}

Let $\mathscr{C}$ be the space of convex bodies in the $d$-dimensional euclidean space $E^{d}$, endowed with the Hausdorff metric. In recent years, many properties that hold for most elements of $\mathscr{C}$ in the sense of Baire categories have been found.

For instance, it was shown that most convex bodies are strictly convex and smooth [5]. T. Zamfirescu proved (in his paper [7], which is closely related to our paper) that most closed convex curves in the plane have precisely 3 points of contact both with their inscribed and with their circumscribed circles. P. Gruber showed in [3] that most convex bodies of $E^{d}$ touch the boundary of both their minimal circumscribed and their maximal inscribed ellipsoids in precisely $d(d+3) / 2$ points. Many other applications of the Baire category theorem in convexity can be found in [4] and [8].

It has been proved (see [1]) that in $E^{d}$ the minimal shell $K(C)$ associated with a convex body $C$ is unique and that on each of the spheres bounding $K(C)$ there are at least 2 points on the boundary of $C$ (for $d=3$ see [6]).

In this note we prove that most convex bodies in $E^{d}$ touch the boundary of their minimal shell in precisely $d+2$ points and that these points form an irreducible set for the minimal shell. The case $d=2$ was treated in [9].

\section{NOTATIONS AND TERMINOLOGY}

We denote by $\mathscr{C}$ the space of convex bodies in $E^{d}$, i.e. the space of all compact convex subsets of $E^{d}$ with nonempty interior, endowed with the Hausdorff metric.

We say that a property holds for most elements of some Baire space, or equivalently that it is generic, if the set of those elements which do not have this property is of first Baire category, i.e. a countable union of nowhere dense sets.

Received by the editors July 10, 1989

1980 Mathematics Subject Classification (1985 Revision). Primary 52A10, 54E52.

Key words and phrases. Convex body, minimal shell, Baire category. 
Let $K$ be the set of points $p$ with

$$
\rho_{0} \leq\left\|p-x_{0}\right\| \leq \sigma_{0},
$$

where $\left\|p-x_{0}\right\|$ is the Euclidean distance of $p$ from a fixed center $x_{0}$. We say that $K$ is a shell of center $x_{0}$ and of radii $\sigma_{0}$ and $\rho_{0}$, bounded by spheres $A$ and $a$ of a center $x_{0}$ and of radii $\sigma_{0}$ and $\rho_{0}$, respectively.

Now we recall the definition of the minimal shell associated with a convex body $C$. In case $d=2$, the minimal shell is called minimal annulus.

Let $B(y, \alpha)=\left\{p \in E^{d}:\|p-y\|<\alpha\right\}$.

For $x \in C$ let

$$
\begin{aligned}
& \sigma(x)=\min \{r: B(x, r) \supseteq C\}, \\
& \rho(x)=\max \{r: B(x, r) \subseteq C\} .
\end{aligned}
$$

The function $\sigma-\rho$ attains its minimal value in a unique point $c \in C$ ([1]; for $d=2$ see [2]). Let $A(x)$ be the boundary of $B(x, \sigma(x))$ and let $a(x)$ be the boundary of $B(x, \rho(x))$. The minimal shell associated with $C$ is the shell $K(C)$ of center $c$ and of radii $\sigma(c)$ and $\rho(c)$. We shall write $c(C)=c$, $A(C)=A(c)$, and $a(C)=a(c)$.

Now let $K$ be a shell as above, of center $x_{0}$ and of radii $\sigma_{0}$ and $\rho_{0}$. Let $p_{1}, p_{2}, \ldots, p_{k}$ be points in the sphere $A$ and let $q_{1}, q_{2}, \ldots, q_{h}$ be points in the sphere $a$. We then say that the sets

$$
\left\{p_{1}, p_{2}, \ldots, p_{k}\right\} \text { and }\left\{q_{1}, q_{2}, \ldots, q_{h}\right\}
$$

cannot be separated if

$$
\operatorname{conv}\left\{p_{1}^{\prime}, p_{2}^{\prime}, \ldots, p_{k}^{\prime}\right\} \cap \operatorname{conv}\left\{q_{1}^{\prime}, q_{2}^{\prime}, \ldots, q_{h}^{\prime}\right\} \neq \varnothing
$$

Here

$$
\begin{aligned}
& p_{i}^{\prime}=x_{0}+\frac{p_{i}-x_{0}}{\sigma_{0}} \quad(i=1,2, \ldots, k), \\
& q_{j}^{\prime}=x_{0}+\frac{q_{j}-x_{0}}{\rho_{0}} \quad(j=1,2, \ldots, h),
\end{aligned}
$$

and $\operatorname{conv}(I)$ denotes the convex hull of the set $I$, i.e. the smallest convex set containing $I$.

The set $\left\{p_{1}, p_{2}, \ldots, p_{k}, q_{1}, q_{2}, \ldots, q_{h}\right\}$ is called irreducible for $K$ if, upon omitting any $p_{i}$ or any $q_{j}$, the remaining set no longer satisfies the previous condition (1).

Notations. The abbreviations bd, int, rel int, and conv stand for boundary, interior, relative interior, and convex hull.

We shall need the following result (see Theorem 2 of [1] or [6] if $d=3$ ) :

(A) The point $x_{0} \in C$ is the center $c(C)$ of the minimal shell of $C$ if and only if there are points $p_{1}, p_{2}, \ldots, p_{k} \in A\left(x_{0}\right) \cap \operatorname{bd} C$ and $q_{1}, q_{2}, \ldots, q_{h} \in$ $a\left(x_{0}\right) \cap$ bd $C$ which cannot be separated. 


\section{MAIN RESUlt}

Theorem. Most convex bodies $C$ in $\mathscr{C}$ have exactly $d+2$ points in common with the boundary of their minimal shell $K(C)$. These points form an irreducible set for $K(C)$.

Proof. First we consider the set

$\mathscr{B}=\{C \in \mathscr{C}:$ bd $C \cap \operatorname{bd} K(C)$ is an irreducible set for $K(C)$ consisting of exactly $d+2$ points $\}$.

We show that $\mathscr{B}$ is dense in $\mathscr{C}$.

Let $D$ be an element of $\mathscr{C}$ and $P$ be a polytope approximating $D$. We set

$$
\begin{aligned}
& \left\{p_{1}, p_{2}, \ldots, p_{m}\right\}=A(P) \cap \operatorname{bd} P \\
& \left\{q_{1}, q_{2}, \ldots, q_{n}\right\}=a(P) \cap \operatorname{bd} P,
\end{aligned}
$$

$c(P)=x_{0}, A(P)=\mathrm{bd} B\left(x_{0}, \sigma_{0}\right)$, and $a(P)=$ bd $B\left(x_{0}, \rho_{0}\right)$. From (A) it follows that $\left\{p_{1}, p_{2}, \ldots, p_{m}\right\}$ and $\left\{q_{1}, q_{2}, \ldots, q_{n}\right\}$ cannot be separated.

Moreover it can be shown (after reindexing if necessary) that there is a subset $\left\{p_{1}, p_{2}, \ldots, p_{k}\right\}$ of $\left\{p_{1}, p_{2}, \ldots, p_{m}\right\}$ and a subset $\left\{q_{1}, q_{2}, \ldots, q_{h}\right\}$ of $\left\{q_{1}, q_{2}, \ldots, q_{n}\right\}$ such that $\left\{p_{1}, p_{2}, \ldots, p_{k}, q_{1}, q_{2}, \ldots, q_{h}\right\}$ is irreducible for $K(P)$ and $4 \leq k+h \leq d+2$.

First we assume that $k+h=d+2$.

From $P$ we obtain another polytope $P^{\prime}$ by cutting the vertices $p_{i} \quad(i=$ $k+1, \ldots, m)$ in such a way that bd $P^{\prime}$ is always a subset of $K(P)$. Next, at each $q_{j}(j=h+1, \ldots, n)$ we construct a sphere $\Sigma_{j}$ externally tangent to $a(P)$ and of diameter so small that the boundary of the convex hull $F$ of $P^{\prime} \cup \Sigma_{h+1} \cup \cdots \cup \Sigma_{n}$ is a subset of $K(P)$ and $a(P) \cap \operatorname{bd} F=\left\{q_{1}, q_{2}, \ldots, q_{h}\right\}$.

Now let

$$
P(\lambda)=(1-\lambda) P+\lambda F \quad \text { where } \lambda \in(0,1) .
$$

It is easy to see that bd $P(\lambda)$ is a subset of $K(P)$. Moreover

$$
\left\{p_{1}, p_{2}, \ldots, p_{k}\right\}=A(P) \cap \mathrm{bd} P(\lambda)
$$

and

$$
\left\{q_{1}, q_{2}, \ldots, q_{h}\right\}=a(P) \cap \mathrm{bd} P(\lambda)
$$

cannot be separated. Then, by $(\mathrm{A})$, we have $K(P(\lambda))=K(P)$.

Therefore $P(\lambda) \in \mathscr{B}$. For $\lambda$ sufficiently small, $P(\lambda)$ is sufficiently close to $P$. Since we can choose $P$ sufficiently close to $D, P(\lambda)$ is also sufficiently close to $D$.

Now let $k+h<d+2$. We treat this case inductively; i.e. we find, close to $P$, another convex body $P(\lambda)$ such that $K(P(\lambda)) \cap$ bd $P(\lambda)$ is an irreducible set for $K(P(\lambda))$ of $k+h+1$ elements.

Assume that $p_{1}$ does not belong to any hyperplane tangent to $a(P)$ at any of the points $q_{1}, q_{2}, \ldots, q_{h}$. By slightly modifying, if necessary, the polytope 
$P$, we can do this in any case. For example, we can construct the following polytope $P^{*}$ close to it: Let $v_{1}, v_{2}, \ldots, v_{m}$ be the points of $B\left(x_{0}, \sigma_{0}-\varepsilon\right)$ nearest to $p_{1}, p_{2}, \ldots, p_{m}$ respectively and $P_{1}=P \cap B\left(x_{0}, \sigma_{0}-2 \varepsilon\right)$. Then we simply put $P^{*}=\operatorname{conv}\left\{P_{1} \cup\left\{v_{1}\right\} \cup\left\{v_{2}\right\} \cup \cdots \cup\left\{v_{m}\right\}\right\}$.

We claim that since the set

$$
\left\{p_{1}, p_{2}, \ldots, p_{k}, q_{1}, q_{2}, \ldots, q_{h}\right\}
$$

is irreducible for $K(P)$, we can choose two points $r$ and $s$ near to $p_{1}$ such that

$$
\left\{r, s, p_{2}, \ldots, p_{k}, q_{1}, q_{2}, \ldots, q_{h}\right\}
$$

is also an irreducible set for $K(P)$.

To prove the above claim, we proceed as follows.

Since $\left\{p_{1}, p_{2}, \ldots, p_{k}, q_{1}, q_{2}, \ldots, q_{h}\right\}$ is an irreducible set for $K(P)$,

$$
\operatorname{conv}\left\{p_{1}^{\prime}, p_{2}^{\prime}, \ldots, p_{k}^{\prime}\right\} \cap \operatorname{conv}\left\{q_{1}^{\prime}, q_{2}^{\prime}, \ldots, q_{h}^{\prime}\right\}
$$

is a point $t$ such that

$$
t \in \operatorname{rel} \text { int } \operatorname{conv}\left\{p_{1}^{\prime}, p_{2}^{\prime}, \ldots, p_{k}^{\prime}\right\} \cap \text { rel int } \operatorname{conv}\left\{q_{1}^{\prime}, q_{2}^{\prime}, \ldots, q_{h}^{\prime}\right\} .
$$

We choose a point $p_{0} \in \operatorname{rel}$ int $\operatorname{conv}\left\{p_{1}^{\prime}, p_{2}^{\prime}, \ldots, p_{k}^{\prime}\right\}$ near to $p_{1}^{\prime}$ in such a way that

$$
t \in \text { rel int } \operatorname{conv}\left\{p_{0}, p_{2}^{\prime}, \ldots, p_{k}^{\prime}\right\} .
$$

If $\pi$ is the hyperplane which contains $p_{0}$ and which is orthogonal to $p_{1}^{\prime}-x_{0}$, we choose a point $r^{\prime}$ of $\pi$, belonging also to $\Sigma=\left\{x \in E^{d}:\left\|x-x_{0}\right\|=1\right\}$, but not to the linear manifold $\mathscr{L}$ spanned by $p_{1}^{\prime}, p_{2}^{\prime}, \ldots, p_{k}^{\prime}, q_{1}^{\prime}, q_{2}^{\prime}, \ldots, q_{h}^{\prime}$. Let us observe that $\mathscr{L}$ is a properly submanifold of $E^{d}$, even if $k+h=d+1$, since $p_{1}^{\prime}, p_{2}^{\prime}, \ldots, p_{k}^{\prime}, q_{1}^{\prime}, q_{2}^{\prime}, \ldots, q_{h}^{\prime}$ are linearly dependent. The straight line $r^{\prime} p_{0}$ meets $\Sigma$ in a point $s^{\prime}$ different from $r^{\prime}$ and not belonging to $\mathscr{L}$. When $p_{0}$ approaches $p_{1}^{\prime}, r^{\prime}$ and $s^{\prime}$ also approach $p_{1}^{\prime}$. Moreover if $p_{0}$ is sufficiently close to $p_{1}^{\prime}$,

$$
t \in \operatorname{rel} \operatorname{int} \operatorname{conv}\left\{r^{\prime}, s^{\prime}, p_{2}^{\prime}, \ldots, p_{k}^{\prime}\right\} .
$$

Then if $r$ and $s$ are the points of $A(P)$ corresponding to $r^{\prime}$ and $s^{\prime}$, we have that the sets of points

$$
\left\{r, s, p_{2}, \ldots, p_{k}\right\} \quad\left\{q_{1}, q_{2}, \ldots, q_{h}\right\}
$$

cannot be separated. Moreover it is possible to prove that

$$
\left\{r, s, p_{2}, \ldots, p_{k}, q_{1}, q_{2}, \ldots, q_{h}\right\}
$$

is an irreducible set for $K(P)$.

Now let $Q=\operatorname{conv}\{P \cup\{r\} \cup\{s\}\}$, where $r$ and $s$ are so close to $p_{1}$ that $a(P) \cap$ bd $Q$ contains $\left\{q_{1}, q_{2}, \ldots, q_{h}\right\}$.

By suitably cutting the vertices $p_{i}(i=1 ; k+1, \ldots, m)$ of $Q$, we obtain a convex $Q^{\prime}$ whose minimal shell is always $K(P)$. As before, at each $q_{j} \quad(j=$ 
$h+1, \ldots, n)$ we construct a sphere $\Sigma_{j}$ of diameter so small that $a(P) \cap \mathrm{bd} F=$ $\left\{q_{1}, q_{2}, \ldots, q_{h}\right\}$ where $F=\operatorname{conv}\left\{Q^{\prime} \cup \Sigma_{h+1} \cup \cdots \cup \Sigma_{n}\right\}$.

If $P(\lambda)=(1-\lambda) Q+\lambda F$ with $\lambda \in(0,1)$ sufficiently small, $P(\lambda)$ is sufficiently close to $Q$. But $Q$ can be constructed sufficiently close to $P$, and therefore $P(\lambda)$ is sufficiently close to $P$. Since, from $(\mathrm{A}), K(P(\lambda))=K(P)$ and since $K(P(\lambda)) \cap \operatorname{bd} P(\lambda)$ is an irreducible set for $K(P(\lambda))$ of $k+h+1$ elements, we see that $P(\lambda)$ is the convex body for which we are looking.

It follows that $\mathscr{B}$ is dense in $\mathscr{C}$.

Now let

$\mathscr{C}(0)=\{C \in \mathscr{C}:$ bd $C \cap$ bd $K(C)$ contains an irreducible set for $K(C)$ of less than $d+2$ points $\}$.

We shall prove that

$$
\mathscr{C}(0) \text { is nowhere dense in } \mathscr{C} \text {. }
$$

The first step is to prove that $\mathscr{C}(0)$ is closed; i.e. if

$$
C_{1}, C_{2}, \cdots \in \mathscr{C}(0) \text { and } \lim _{n \rightarrow \infty} C_{n}=C \text {, }
$$

then $C \in \mathscr{C}(0)$. By the definition of $\mathscr{C}(0)$ there exists an irreducible set for $K\left(C_{i}\right)$

$$
\left\{p_{i 1}, p_{i 2}, \ldots, p_{i k_{i}}, q_{i 1}, q_{i 2}, \ldots, q_{i h_{i}}\right\},
$$

where $k_{i}+h_{i}<d+2$.

By considering a suitable subsequence and reindexing if necessary, we may assume that $k_{1}=k_{2}=\cdots=k$ and $h_{1}=h_{2}=\cdots=h$, say, with $k+h<d+2$ and

$$
\begin{aligned}
& \lim _{n \rightarrow \infty} p_{n j}=p_{j} \quad(j=1,2, \ldots, k), \\
& \lim _{n \rightarrow \infty} q_{n j}=q_{j} \quad(j=1,2, \ldots, h) .
\end{aligned}
$$

A routine argument together with (A) shows that if

$$
C_{1}, C_{2}, \cdots \in \mathscr{C}(0) \text { and } \lim _{n \rightarrow \infty} C_{n}=C \text {, }
$$

then

$$
\lim _{n \rightarrow \infty} K\left(C_{n}\right)=K(C)
$$

Thus $p_{1}, p_{2}, \ldots, p_{k}, q_{1}, q_{2}, \ldots, q_{h} \in \operatorname{bd} C \cap \mathrm{bd} K(C)$.

Since

$$
\left\{p_{i 1}, p_{i 2}, \ldots, p_{i k}\right\},\left\{q_{i 1}, q_{i 2}, \ldots, q_{i h}\right\}
$$

cannot be separated, we have that

$$
\left\{p_{1}, p_{2}, \ldots, p_{k}\right\},\left\{q_{1}, q_{2}, \ldots, q_{h}\right\}
$$

cannot be separated, with $k+h<d+2$. 
From these sets we can choose a subset irreducible for $K(C)$. It follows that $\mathscr{C}(0)$ is closed in $\mathscr{C}$. By (2) int $\mathscr{C}(0)=\varnothing$, and this concludes the proof of (3).

For $i \in\{1,2, \ldots\}$ let $\mathscr{C}(k, i)=\{C \in \mathscr{C}:$ bd $C \cap$ bd $K(C)$ contains at least $d+3$ points, $k$ of which are elements of $A(C)$, i.e.

$$
\text { bd } C \cap \operatorname{bd} K(C) \supseteq\left\{p_{1}, p_{2}, \ldots, p_{k}, q_{k+1}, \ldots, q_{d+3}\right\},
$$

with $p_{1}, p_{2}, \ldots, p_{k} \in A(C), q_{k+1}, \ldots, q_{d+3} \in a(C)$, and moreover $\left\|p_{r}-p_{s}\right\| \geq$ $\left.1 / i,\left\|q_{m}-q_{n}\right\| \geq 1 / i\right\}$.

An elementary compactness argument using (4) shows that $\mathscr{C}(k, i)$ is closed in $\mathscr{C}$. By $(2) \mathscr{C}(k, i)$ has empty interior and thus, being closed, is nowhere dense in $\mathscr{C}$.

Now if $\mathscr{C}(i)=\{C \in \mathscr{C}:$ bd $C \cap$ bd $K(C)$ contains at least $d+3$ points such that if $p_{r}, p_{s} \in A(C) \cap \mathrm{bd} C$ and $q_{m}, q_{n} \in a(C) \cap \operatorname{bd} C$ then $\left\|p_{r}-p_{s}\right\| \geq 1 / i$ and $\left.\left\|q_{m}-q_{n}\right\| \geq 1 / i\right\}$, then

$$
\mathscr{C}(i)=\bigcup_{k} \mathscr{C}(k, i)
$$

Hence

$$
\bigcup_{i=1}^{\infty} \mathscr{C}(i)=\{C \in \mathscr{C}: \text { bd } C \cap \text { bd } K(C) \text { contains at least } d+3 \text { points }\}
$$

is a countable union of nowhere dense sets of $\mathscr{C}$. Thus $\mathscr{C}(0) \cup \bigcup_{i=1}^{\infty} \mathscr{C}(i)$ is also a countable union of nowhere dense sets of $\mathscr{C}$. This completes the proof.

\section{REFERENCES}

1. I. Bárány, On the minimal ring containing the boundary of a convex body, Acta Sci. Math (Szeged) 52 (1988), 93-100.

2. T. Bonnesen, Les problèmes des isopérimètres et des isépiphanes, Gauthier-Villars, Paris, 1929.

3. P. Gruber, Minimal ellipsoids and their duals, Rend. Circ. Mat. Palermo 37 (1988), 35-64.

4. $\_$, Results of Baire category type in convexity, Ann. New York Acad. Sci. 440 (1985), 163-169.

5. V. Klee, Some new results on smoothness and rotundity in normed linear spaces, Math. Ann. 139 (1959), 51-63.

6. N. Kritikos, Über Konvexe Flächen und einschliessende Kugeln, Math. Ann. 96 (1927), 583-586.

7. T. Zamfirescu, Inscribed and circumscribed circles to convex curves, Proc. Amer. Math. Soc. 80 (1980), 455-457.

8. __ Using Baire categories in geometry, Rend. Sem. Mat. Univ. Politecn. Torino 43 (1985), 67-88.

9. A. Zucco, Minimal annulus of a convex body, Arch. Math. 52 (1989), 92-94.

Dipartimento di Matematica, Università di Torino, 10123 Torino, Italy 